Keywords: Nationwide cross-sectional survey, Medication, Psychotherapy, Children, Adolescents

\title{
Psychiatric treatments for children and adolescents preferred by spanish psychiatrists
}

Josep Toro ${ }^{\star \star \star *}$
María Mur*
Tomás Cantó

* Dept. Child and Adolescent Psiquiatry an Psychology Institut Clinic de Neurociències, Hospital Clinic

** Dept. Psiquiatry and Clinical

Psichobiology

Facultat de Medicina,Universitat de Barcelona

*** Unidad de Salud Mental de Alicante

SPAIN

ABSTRACT - Objectives: To study the prescription criteria of Spanish psychiatrists treating children and adolescents.

Methods: a survey was designed to record their first choice and complementary preferences for pharmacological, psychotherapeutic and psychoeducational interventions in five disorders: autism, depression, separation anxiety, obsessive compulsive and attentiondeficit/hyperactivity disorders.

Results: One hundred and nine psychiatrists responded. No distinction was made between children and adolescents. Around $90 \%$ recommended all three types of intervention in the five disorders. Only 2-10\% would use only one treatment. Antidepressants were the most frequently prescribed drugs (recommended by $58 \%$ ), followed by anxiolytics $(33 \%)$, antipsychotics $(24 \%)$, stimulants $(20 \%)$, beta-blockers $(19 \%)$, mood stabilizers (10\%) and alpha-adrenergics (4\%). Cognitive-behavioral therapy was the most popular approach, recommended by $66 \%$; a third of the interviewees recommended family, support, interpersonal and dynamic psychotherapy. Interestingly, respondents quite frequently prescribe drugs, drug combinations and psychotherapies whose efficacy has not been demonstrated in the disorders in question.

Conclusions: The majority of Spanish psychiatrists preferred the combined treatments in all disorders. There seems to be a tendency towards excessive generalization of therapeutic results obtained in adults. 


\section{Introduction}

The last twenty years have seen radical changes in therapeutic strategies in psychiatry. The advances in diagnostic criteria, in assessment procedures and in understanding of mental illness, and above all the development of psychopharmacology, have opened up new therapeutic perspectives that were unimaginable only a few years ago. Established psychotherapeutic interventions (the psychodynamic, cognitive-behavioral and systemic approaches) have changed little, but clinical psychopharmacology, which was practically non-existent in children and adolescents a few years ago, is now experiencing a real boom. In only a few years the handbooks of child psychopharmacology have multiplied. General psychiatry publications show a continuous increase in articles on the subject, and in the last ten years two specialist international publications have been launched, the Journal of Child and Adolescent Psychopharmacology and Child and Adolescent Psychopharmacology News.

At a time of such rapid change in therapeutic strategies, the attitudes and behaviors of child psychiatry professionals merit close study. In the literature the few studies of this kind that have been published usually refer to the prescription of psychotropic drugs (frequently of a single type) and ignore psychosocial interventions.

It is generally believed that the use of psychotropic drugs in minors is on the increase, but very few studies have set out to determine whether this is actually the case. Sourander et al. ${ }^{1}$, in Finland, compiled information on the pharmacological treatment administered to hospitalized children and adolescents. Forty-three percent had scheduled medication and $28 \%$ "had required" medication. In comparison with a study conducted in 1988, those authors reported an increase in the prescription of psychotropic drugs, principally antidepressants. In Baltimore County, USA, between 1988 and 1994 there was an increase in overall psychotropic medication treatment, multiple concurrent medication treatments (polypharmacy), and antidepressant treatment with serotonin reuptake inhibitors ${ }^{2}$. Another study from the US found that stimulant therapy within ADHD youth visits rose from $62.6 \%$ in 1989 to $76.6 \%$ in $1996^{3}$. A literature review highlighted the increase in use and number of psychotropic drugs even in preschool children ${ }^{4}$. However, prescription patterns vary from country to country. British child psychiatrists, for example, tended to use antidepressants far less than their counterparts in the US ${ }^{5}$.

Very few studies compare the use of different therapeutic procedures. A Danish study, which included all current inpatients in spring 1990, found that primary caretaking was the main treatment method (100\%), and individual psychotherapy and family therapy were each offered to one third of subjects ${ }^{6}$. Eight per cent were medicated, $6 \%$ with psychoactive drugs.

In this study we interviewed a sample of Spanish child and adolescent psychiatrists to determine their preferences and recommendations for psychopharmacological and psychosocial treatments. They were asked to express their opinions taking account of: 1) all the possible therapeutic procedures; 2) five distinct, significant psychiatric disorders in children and adolescents; and 3) patients' stage of development. 


\section{Method}

\section{Survey}

A survey was designed in which psychiatrists had to respond to the following question: Which treatments do you consider to be first choice, and which do you consider complementary, for each disorder and age group? Interviewees were asked to score first choice treatments as " 1 " and complementary treatments as " 2 ". The ten treatments were: psychotherapies, orientation/ counseling and psychoeducation, antidepressants, neuroleptics, anxiolytics, mood stabilizers, psychostimulants, alpha-adrenergic agonists, beta-adrenergic blockers, and others. Each type included various components so the respondent could choose from between 45 therapeutic procedures. Respondents had to record their preferred procedures (either first choice or complementary) for children and adolescents with the following disorders: autism and other pervasive development disorders, depressive disorder, separation anxiety disorder, obsessive compulsive disorder, and attention-deficit/hyperactivity disorder. The survey was accompanied by a questionnaire in which respondents recorded their sociodemographic data and stated their professional activity.

It was difficult to decide on the target population for the survey. The study focused on specialists in children and adolescents, but in Spain this professional qualification does not yet exist and a number of psychiatrists treat both children and adults. An extra difficulty is the fact that each region of Spain has its own organizational structure for the psychiatric care of children and adolescents. This means that there is no official record of child and adolescent psychiatrists. To give a rough idea of their numbers in
Spain, the AEPIJ, the Spanish Association of Child and Adolescent Psychiatry, had 238 members at the time of the survey (but many of them visit adults too). We decided to use the profiles of psychiatrists in the possession of a pharmaceutical company's sales representatives. In 2002 these representatives were asked to present psychiatrists a survey accompanied by a letter signed by the first author and by the President of the AEPIJ describing the study and requesting their cooperation. Respondents also received a stamped addressed envelope in which to return the survey. Because of these logical problems we could not be sure that the distribution was complete or homogeneous, or that the correct respondents had been contacted. An added problem was the fact that the survey was administered by a representative of a pharmaceutical firm and included items on psychotropic drugs, a coincidence that may well have caused respondents to wonder about possible commercial interests, in spite of the fact that the letter of presentation bore the letterheads of the University of Barcelona and the AEPIJ. In any case, 953 surveys were prepared for distribution among psychiatrists all over Spain. Several of these surveys did not reach the intended respondents and others were delivered to psychiatrists who do not work with children and therefore did not respond, but we do not know how many.

One hundred and nine valid surveys were returned, $11.4 \%$ of those sent out. For the reasons just mentioned, this figure by no means represents the total number of child psychiatrists in Spain. However, it does represent around $50 \%$ of the members of AEPIJ, the specialists most directly involved in the issues raised by the survey. Seven respondents answered only the questions referring to adolescents, so 102 surveys referred to children. 


\section{Results}

\section{The respondents}

Among the respondents, $8.3 \%$ worked only in private practice and $60.6 \%$ worked only in a public center. Only $59.8 \%$ worked exclusively in child psychiatry and $62.6 \%$ reported practicing general psychiatry in one way or other. They did not show statistically significant differences in their responses; therefore we consider them as a only group. Most attended patients at a mental health center $(49.5 \%)$ or a general hospital (34.3\%). Most (55.6\%) had seen more than thirty different patients in the previous month: the rest had seen fewer.

None of the variables analyzed indicated whether the therapeutic recommendations were aimed at children or at adolescents. As a result, the data presented correspond to the sum of the two groups.

\section{General treatment procedures}

In psychiatric treatment for minors, whatever their disorder, the great majority of respondents (84-99\%) consider psychotherapeutic, pharmacological and psychoeducational interventions to be necessary either as first choice or complementary treatments (Table I). More than $98 \%$ of psychiatrists judged psychotherapy to be necessary in depression, separation anxiety and OCD; more than $94 \%$ considered pharmacological treatment to be necessary in depression, OCD, ADHD and autism; and more than $94 \%$ thought psychoeducational interventions necessary in autism and ADHD.

Table I

Percentages of psychiatrists considering psychotherapy, psychotropic drugs or psychoeducational intervention as first choice or complementary in the treatment of 5 psychiatric disorders.

\begin{tabular}{lccccc} 
& Autism & Depression & Sep. Anxiety & OCD & ADHD \\
\hline Psychotherapy & 81.7 & 99.1 & 99.1 & 98.2 & 88.1 \\
Psychotropic drugs & 94.5 & 97.3 & 89 & 97.2 & 94.5 \\
Psychoeducation & 95.5 & 88.1 & 87.2 & 84.4 & 94.5 \\
\hline
\end{tabular}

Cognitive-behavioral therapy was the most favored approach, recommended by $66.2 \%$ of the sample, followed by family $(37.6 \%)$, support $(34.5 \%)$, interpersonal $(33.7 \%)$ and dynamic $(32.4 \%)$ psychotherapies..

Overall, the most recommended drugs were antidepressants (58.5\%), followed by anxiolytics $(32.6 \%)$, antipsychotics $(24 \%)$, stimulants (19.6\%), beta-blockers (19.3\%), mood stabilizers $(10.4 \%)$ and alpha-adrenergics $(3.8 \%)$.

\section{Autism}

Almost half the sample (47\%) considered the association of psychotropic drugs and psychotherapy as first choice, but in the other half the opinions ranged widely; $12 \%$ would prescribe drugs alone and $3 \%$ would use only psychotherapy (though they acknowledge the need for psychoeducational interventions) (Table II).

Around $45 \%$ prefer family, cognitivebehavioral or support psychotherapy, and around 22\% dynamic therapy (Table III); 
these figures indicate that a substantial number of psychiatrists recommend more than one type of psychotherapy simultaneously.

Antipsychotics were the drugs of choice for $70 \%$ of respondents, but all types of drug were considered first choice treatment by one or more (Table IV). However, with the exception of anxiolytics (considered first choice by more than 25\%), psychotropic drugs tended to be considered as complementary.

Table II

Percentages of recomended combinations of psychotropic drugs and psychotherapy*.

\begin{tabular}{lccccr} 
& Autism & Depression & Sep. Anxiety & OCD & ADHD \\
\hline 1 Drug, 1 Psy & 47.7 & 84.4 & 68.8 & 84.4 & 65.1 \\
1 Drug, 2 Psy & 9.2 & 9.2 & 10.1 & 10.1 & 14.6 \\
1 Psy, 2 Drug & 15.6 & 2.7 & 10.1 & 0.0 & 2.7 \\
Only Drug & 12.8 & 0.9 & 0.0 & 1.8 & 11.0 \\
Only Psy & 3.7 & 2.7 & 10.1 & 2.7 & 4.6 \\
\hline
\end{tabular}

* 1: First choice; 2: Complementary; Drug: Psychotropic drugs; Psy: Psychotherapy

Table III

Percentages of recomended psychotherapies (first choice and complementary).

\begin{tabular}{lccccc} 
& Autism & Depression & Sep. Anxiety & OCD & ADHD \\
\hline Cog. Behav. & 45.9 & 67.0 & 67.9 & 87.2 & 63.3 \\
Dynamic & 22.9 & 41.3 & 38.6 & 33.9 & 25.7 \\
Family & 46.7 & 38.5 & 39.4 & 25.7 & 38.6 \\
Interpersonal & 20.1 & 49.5 & 34.9 & 33.0 & 31.2 \\
Support & 43.2 & 37.6 & 25.7 & 33.0 & 33.0 \\
\hline
\end{tabular}

Table IV

Percentages of considered as first choice (in bold type) or complementary psychotropic drugs.

\begin{tabular}{lccccc} 
& Autism & Depression & Sep. Anxiety & OCD & ADHD \\
\hline Antidepressants & $13.8-25.7$ & $94.5-2.8$ & $65.1-11.0$ & $96.3-0.9$ & $22.9-32.1$ \\
Anxyolitics & $25.7-28.4$ & $32.1-41.3$ & $56.9-27.5$ & $35.8-39.4$ & $12.8-18.3$ \\
Antipsychotics & $69.7-24.8$ & $8.3-18.3$ & $6.4-8.3$ & $18.3-36.7$ & $17.4-35.8$ \\
Mood stabilizers & $15.6-25.7$ & $17.4-27.5$ & $3.7-10.1$ & $6.4-11.0$ & $9.2-17.4$ \\
Stimulants & $5.5-16.5$ & $5.5-16.5$ & $0.9-8.3$ & $1.8-8.3$ & $85.3-4.6$ \\
$\alpha$-adrenergics & $5.5-16.5$ & $1.8-7.3$ & $1.8-7.3$ & $0.9-10.1$ & $9.2-17.4$ \\
$\beta$-blockers & $4.6-10.1$ & $2.8-8.3$ & $6.4-8.3$ & $1.8-10.1$ & $3.7-6.4$ \\
\hline
\end{tabular}

\section{Depression}

Eighty-four per cent of respondents considered the simultaneous application of psychotropic drugs and psychotherapy to be first choice in child and adolescent depression. For $12.5 \%$, drugs were not first choice (Table II). Only $0.9 \%$ believed that psy- chotherapy alone should be used to treat depression.

Cognitive-behavioral psychotherapy was the most recommended approach (67\%), followed by interpersonal psychotherapy $(49.5 \%)$. The other types scored around $40 \%$ (Table III). 
Antidepressants were recommended as first choice drugs by $94.5 \%$, followed at a considerable distance by anxiolytics (32\%) (Table IV). The latter were considered first choice or complementary by $73 \%$, suggesting that most respondents advocated simultaneous prescription of antidepressants and anxiolytics for depression. Antipsychotic drugs were used by $26.6 \%$ of psychiatrists.

\section{Separation anxiety disorder}

A significant majority (68.8\%) favored the simultaneous use of psychotropic drugs and psychotherapy (Table II). Ten per cent prescribed psychotherapy alone, and none drugs alone.

Cognitive-behavioral psychotherapy was by a long way the most favored type of treatment $(67.9 \%)$, while family, dynamic and interpersonal psychotherapies were supported by around 35\% (Table III).

Antidepressants were the first choice drugs for $65.1 \%$ and anxiolytics for $56.9 \%$ (Table IV). However, adding together first choice and complementary treatments, anxiolytics were the most recommended, reaching $84.4 \%$. Beta-blockers and antipsychotics with total scores of $19.2 \%$ and $14.7 \%$ respectively, were also popular.

\section{Obsessive-compulsive disorder}

The vast majority $(84.4 \%)$ recommended simultaneous prescription of psychotropic drugs and psychotherapy in OCD (Table II). Psychotropic drugs were considered first choice, with or without psychotherapy, by $96.3 \%$ of respondents. Only $2.75 \%$ advocated psychotherapy alone.

Cognitive-behavioral therapy was the most recommended approach $(87.2 \%)$, fol- lowed at a long distance by dynamic, support and interpersonal psychotherapies, all of which were recommended by $33 \%$ of the sample (Table III).

Antidepressants were considered first choice by almost all respondents $(96.3 \%)$; in second place were anxiolytics (35.8\%), though counting both first choice and complementary treatments anxiolytics were recommended by $75.2 \%$ (Table IV). Interestingly, ten per cent considered alpha-adrenergic agonists and beta-blockers complementary in OCD treatment.

\section{Attention deficit/hyperactivity disorder}

In ADHD combined treatment of drugs and psychotherapy was chosen by the majority $(65.13 \%)$, but $90.7 \%$ considered the prescription of a drug as first choice (Table II). Eleven per cent would prescribe psychotherapy of some kind and $4.5 \%$ would not use drugs.

Sixty-three per cent of psychiatrists use cognitive-behavioral therapy and around $35 \%$ family, support and interpersonal psychotherapies (Table III). A quarter would use dynamic therapy.

Most $(85.3 \%)$ considered stimulants to be first choice; $22.9 \%$ antidepressants, a figure that rises to $55 \%$ when first choice and complementary treatments are added together (Table IV). Fifty-three per cent considered antipsychotics and $31 \%$ anxiolytics.

\section{Discussion}

This is the first study of the prescription criteria used by Spanish psychiatrists in the 
treatment of children and adolescents with psychiatric disorders. As treatment aims, we chose five representative psychopathologies in children and adolescents. Though we sought to distinguish between children and adolescents, none of the interviewees' responses regarding prescriptions and recommendations differentiated significantly between the two age groups, suggesting that no distinction is made according to developmental stage.

The vast majority of respondents consider the use of psychotherapy (82-99\%) and psychotropic drugs (89-97\%) to be necessary in the five disorders and opt for combined treatments. The majority (84-95\%) also recommend psychoeducational interventions. Few studies to date have empirically validated sufficient numbers of single treatments, and even fewer have assessed most combined treatments.

In all the disorders cognitive-behavioral psychotherapies were preferred by most psychiatrists, being twice as popular as other kinds. It is true that cognitive-behavioral treatment has proved more effective in most psychiatric disorders in children and adolescents $^{7}$, but the support of Spanish child psychiatrists for this approach is a recent phenomenon, probably reflecting an increasing preference for evidence-based practices. Until a few years ago psychodynamic therapy predominated, but today it is no more popular than other approaches. In the context of the survey perhaps the term "family psychotherapy" should not be interpreted in the strict sense, but more broadly, as "family intervention". The figure of $33 \%$ for interpersonal therapy is surprising, as it is relatively undeveloped in practice and its efficacy is only proven in adolescent depression. It is possible that some of the interviewees may have lacked a thorough knowledge of the technique and interpreted interpersonal therapy as a psychosocial (or simply social) intervention.

Coinciding with the current controversy on the use of antidepressants in the young, we found that these drugs are the most frequently prescribed by Spanish child psychiatrists, following the trends found in other countries $^{8,1}$. The use of antidepressants in child psychiatry appears to have doubled in the last ten years ${ }^{9}$. In spite of their unreliability in most disorders, anxiolytics were the most recommended drugs after antidepressants, being prescribed by a third of the psychiatrists. For their part, beta-blockers are used by $20 \%$ of psychiatrists, though hardly any trials of these drugs have been carried out in children and adolescents. All this suggests that these drugs are prescribed in this younger population due to their application in adults.

\section{Autism}

Naturally, more than $95 \%$ of respondents consider psychoeducational intervention to be essential in autism.

Practically all the psychiatrists supported the prescription of drugs to patients with autism, particularly antipsychotics, though a quarter considered them to be complementary. Only $68 \%$ considered drugs as first choice. All drug types were considered first choice by substantial numbers of interviewees, reflecting the lack of consensus or reliable criteria due to the fact that the area remains almost entirely unexplored. To date, only one antipsychotic (risperidone), two antidepressants (clomipramine and fluvoxamine) and one a-2-adrenergic agonist (clonidine) have proved effective in reducing certain autistic symptoms in placebocontrolled studies ${ }^{10}$. 
Psychotherapy was recommended by $81 \%$ of psychiatrists, though the type of therapy chosen varied widely. Almost half advocated family interventions and cognitive-behavioral procedures, but around $20 \%$ propose psychodynamic or interpersonal treatments whose efficacy has not been proven to date.

\section{Depression}

Most of the respondents (84\%) consider simultaneous use of drugs and psychotherapy in depression to be fundamental. Indeed this strategy is justified by recent studies ${ }^{11}$. However, even more (94\%) believe that drugs are first choice, with or without psychotherapy. Only $3 \%$ prescribe psychotherapy alone, though most $(86 \%)$ consider it first choice.

Almost all those who favor prescribing psychotropic drugs recommend antidepressants. Interestingly, these drugs are considered favorably for depression in spite of their possible role in increasing suicidal tendencies and in spite of doubts regarding their efficacy ${ }^{12,13}$. Given the lack of supporting evidence, it is surprising that a third of respondents chose an anxiolytic as first choice for depression in minors and a quarter recommended antipsychotics as first choice or complementary. All this suggests a high rate of combined pharmacological treatments whose efficacy is not proven.

To treat depression the interviewees' preferences for the five types of psychotherapy varied, though only cognitive-behavioral and interpersonal therapies have proven therapeutic efficacy ${ }^{14,11}$ and are the most recommended. A large group advocated more than one psychotherapeutic intervention, such as cognitive-behavioral and family therapy.

\section{Separation anxiety disorder}

Almost $70 \%$ of interviewees considered a combination of drugs and psychotherapy as first choice in SAD, though this combination has not been shown to be more effective than either of these treatments alone. But all respondents recommended psychotherapeutic intervention.

More than half the psychiatrists considered the prescription of antidepressants and/or anxiolytics as first choice. The efficacy of SSRI has been demonstrated in various controlled studies, though all of them were performed with samples that mixed SAD and other anxiety disorder ${ }^{15,16}$. Almost $60 \%$ of interviewees considered anxiolytics to be first choice treatment and $85 \%$ as first choice or complementary; these figures are surprising, since their efficacy in SAD has not been demonstrated, and the risk of tolerance and dependence in minors has not been studied to date ${ }^{17}$. No psychiatrists advocated treating SAD with psychotropic drugs alone.

Cognitive-behavioral therapy is the only treatment whose efficacy is supported by data from randomized controlled studies ${ }^{18}$. However, $30 \%$ of the psychiatrists do not recommend it. In SAD, as in other disorders, they often advocate more than one type of psychotherapy.

\section{Obsessive compulsive disorder}

Again, the vast majority (85\%) consider drugs and psychotherapy first choice, but drugs were considered first choice by almost all those surveyed. Only 3 (2.7\%) advocated psychotherapy alone. Practically all the participants chose antidepressants, SSRI in the great majority of cases. The efficacy of these drugs, along with cloripramine, has been 
demonstrated in controlled trials ${ }^{19}$; this is not the case of anxiolytics or antipsychotics, recommended by a significant number of psychiatrists.

Almost all respondents recommended cognitive-behavioral therapy. Until recently only open trials had been performed, but now the efficacy of this treatment has been demonstrated by a series of controlled studies including the family ${ }^{20}$. The inclusion of the family, reflecting a psychoeducational orientation, may account for the fact that a quarter of respondents also advocate family psychotherapy. More than a third recommend psychodynamic therapy, in spite of the fact that it is not effective for obsessions and rituals in patients with OCD (though it may contribute to raising compliance with behavior or psychopharmacological therapies) $)^{2}$.

There is no evidence in children and adolescents that antidepressants (SSRI or clomipramine) in association with cognitive-behavioral therapy, the treatment preferred by respondents, is more effective than either of these procedures alone. The probably higher efficacy of combined treatment over the drug alone has only been demonstrated in adults, in whom clomipramine with exposure and ritual prevention has achieved treatment response rates of 70$79 \%$ and clomipramine alone $42-48 \%$; however, behavioral treatment alone achieved $62-86 \% 21$. This means that the drug does not seem to increase the efficacy of behavioral therapy, and so the latter is the treatment of choice.

\section{Attention-deficit/hyperactivity disorder}

Nine out of every ten psychiatrists consider the use of psychotropic drugs in ADHD to be first choice. Psychotherapy is first choice for $7 \%$. Only $4.6 \%$ advocate psychotherapy alone, without the use of drugs. More than 180 placebo-controlled trials have demonstrated the efficacy of stimulants ${ }^{22}$; in spite of this, $15 \%$ of psychiatrists do not consider them to be the drugs of choice. In fact, all types of psychotropic drugs are considered first choice by some psychiatrists. The evidence may justify the use of certain antidepressants, especially if there is no response to stimulants, or a combination of antidepressants and clonidine, an a-adrenergic agonist $^{23}$. But there is no justification for prescribing antipsychotics, anxiolytics or mood stabilizers as first choice medication in ADHD.

For three out of every four psychiatrists psychotherapy is first choice in ADHD and $65 \%$ consider it as important as psychotropic drugs. Only behavior therapy procedures, implemented in the home, classroom, and in specialized environments have a base in evidence $^{24}$. In spite of this many respondents, between 25 and $38 \%$, consider family therapy, support, interpersonal and psychodynamic therapy as first or second line treatment of choice.

\section{Limitations}

We cannot be sure that the survey is representative of all Spanish childhood and adolescence psychiatrists. The percentage of responses is low $(11.4 \%$ of those distributed). Nonetheless it should be borne in mind that many of those who received the questionnaire were not treating children or adolescents and so they had no reason to respond. Another point is that we cannot be sure that the representatives of the pharmaceutical companies gave all the surveys to all the intended recipients; indeed, this is 
unlikely. In spite of this, the number of responses obtained was quite high, representing almost half the membership of the Spanish Association of Child and Adolescent Psychiatry, the association under whose auspices the survey was carried out.

It is also possible that the sample is biased towards those who support the use of pharmacological treatment, because representatives of a pharmaceutical company were used as intermediaries. Respondents who were less inclined to prescribe these treatments may have suspected a commercial interest and may have preferred not to participate.

In spite of these limitations, we believe that the results provide a fairly accurate refection of the current state of attitudes to treatment of children and adolescents among Spanish psychiatrists. Since the survey is the first of its kind in Spain it is impossible to draw conclusions on the evolution of these therapeutic attitudes and practices.

\section{Conclusions}

The vast majority of Spanish psychiatrists who work with children and adolescents consider combined, pharmacological, psychotherapeutic and psychosocial treatments as first choice in all the disorders assessed here. Only a small minority, between 2 and $10 \%$, would only use a single type of treatment in particular disorders.

As expected, antidepressants were the most recommended drugs in depression, separation anxiety disorder and obsessive-compulsive disorder, antipsychotics in autism, and stimulants in ADHD. However, in all the disorders there are psychiatrists who pre- scribe as first choice drugs whose efficacy has not been definitively demonstrated, or in some cases even disproved. An example is the high rate of prescription of anxiolytics.

Cognitive-behavioral psychotherapy was the most popular approach, though respondents often recommend combinations of two or more psychotherapies.

We found that many therapeutic decisions are taken for subjective reasons or by extrapolation of results in adults. More controlled trials are needed to assess the therapeutic efficacy both of many psychotropic drugs and of psychotherapeutic procedures, including combined treatments. It would be interesting to compare these results with those of similar studies in other countries.

\section{Acknowledgment}

We wish to thank Dr. Cobaleda and Glaxo Smith Kline its contribution to spreading the survey.

\section{References}

1. Sourander A, Ellila H, Valimaki M, Aronen ET. Psychopharmacological treatment of child and adolescent psychiatric inpatients in Finland. J Child Adolesc Psychopharmacol 2002; 12: 147-155.

2. Safer DJ. Changing patterns of psychotropic medications prescribed by child psychiatrists in the 1990s. J Child Adolesc Psychopharmacol 1997; 7: 267-274.

3. Zito JM, Saber DJ, dosReis S et al. Psychotherapeutic medication patterns for youths with attention-deficit/ hyperactivity disorder. Arch Pediatr Adolesc Med 1999; 153: $1257-1263$.

4. Minde K. The use of psychotropic medication in preschoolers: some recent developments. Can J Psychiatry 1998; 43: 571-575. 
5. Bramble DJ. Antidepressant prescription by British child psychiatrists: practice and safety issues. J Am Acad Child Adolesc Psychiatry 1995; 34: 327-331.

6. Sogaard HW, Jorgensen OS, Piha J. Child psychiatric in-patient treatment. Ugeskr Laeger 1988; 160: 3383-3387.

7. Barrett PM, Ollendick TH, eds. Interventions that work with children and adolescents. Prevention and treatment. Chichester: Wiley; 2004.

8. Sǿrensen CB, Jepsen EB, Thomsen PH, Dalsgaard S. Indications of the use of antidepressants in child and adolescent psychiatry. Eur Child Adolesc Psychiatry 2003; 12: 114-121.

9. Hunkeler EM, Fireman B, Lee J et al. Trends in use of antidepressants, lithium, and antoconvulsivants in Kaiser Permanente-Insured youths, 1994-2003. J Child Adolesc Psychopharmacol 2005; 15: 26-37.

10. Stigler KA, Posey DJ, McDougle CJ. Advances in the pharmacotherapy of pervasive developmental disorders (PDD). Child Adolesc Psychopharmacol News 2003; 8: 411.

11. Treatment for Adolescents with Depression Study. Fluoxetine, cognitive-behavioral therapy, and their combination for adolescents with depression. JAMA 2004; 292, 807-820.

12. European Medicines Agency. European Medicines Agency finalises review of antidepressants in children and adolescents. 1995. EMEA/CHMP/128918/2005 corr.

13. Toro J, Rey JM. Fármacos antidepresivos en niños y adolescentes. Med Clin 2005; 125: 143-144.

14. Mufson L, Dorta KP, Wickramaratne P et al. A randomized effectiveness trial of interpersonal psychotherapy for depressed adolescents. Arch Gen Psychiatry 2004; 61: 577-584.

15. Birmaher B, Axelson DA, Monk K et al. Fluoxetine for the treatment of childhood anxiety disorders. J Am Acad Child Adolesc Psychiatry 2003; 42: 415-423.

16. Research Unit on Pediatric Psychopharmacology Anxiety Study Group. Fluvoxamine for the treatment of anxiety disorders in children and adolescents. N Engl J Med 2001; 344: 1279-1285.
17. Velosa JF, Riddle MA. Pharmacologic treatment of anxiety disorders in children and adolescents. Child Adolesc Psychiatr Clin N Am 2000; 9: 119-133.

18. Labellarte MJ, Ginsburg GS, Walkupp JT. The treatment of anxiety disorders in children and adolesscents. Biol Psychiatry 1999; 46: 1567-1578.

19. Barrett PM, Healy-Farrell L, Piacentini J, March J. Obsessive-compulsive disorder in childhood and adolescence: Description and treatment. In: Barrett PM, Ollendick TH, (Eds). Interventions that work with children and adolescents. Chichester, England: Wiley; 2004. p. 187216.

20. Barret PM, Healy-Farrell L, March JS. Cognitivebehavioral family treatment of childhood obsessive-compulsive disorder: A controlled trial. J Am Acad Child Adolesc Psychiatry 2004; 43: 46-62.

21. Foa EB, Liebowitz MR, Kozak MJ et al . Randomized, placebo-controlled trial of exposure and ritual prevention, clomipramine, and their combination in the treatment of obsessive-compulsive disorder. Am J Psychiatry 2005; 162: 151-161.

22. Greenhill LL, Ford RE. Child attention-deficit hyperactivity disorder: Pharmacological treatments. In: Nathan PE, Gorman JM, (Eds.) A guide to treatments that work, $2^{\text {nd }}$ edn. New York: Oxford University Press; 2002. p. $25-55$.

23. Hazell PL, Stuart JE. A randomized controlled trial of clonidine added to psychoestimulant medication for hyperactive and aggresive children. J Am Acad Child Adolesc Psychiatry 2003; 42: 886-894.

24. Hinshaw SP, Klein RG, Abikoff HB. Childhood attention-deficit hyperactivity disorder: Nonpharmacological treatments and their combination with medication. In: Nathan PE, Gorman JM, (Eds). A guide to treatments that work, $2^{\text {nd }}$ ed. New York: Oxford University Press; 2002. p. $3-23$.

Address for correspondence:

Josep Toro

Servei de Psiquiatria i Psicologia Infantil i Juvenil

Hospital Clínic

Villarroel 170; 08036 Barcelona

e-mail: jtoro@clinic.ub.es

SPAIN 